\title{
PLANETARY CAMERA OBSERVATIONS OF THE CENTRAL PARSEC OF M32 ${ }^{1}$
}

TOD R. LAUER

Kitt Peak National Observatory, National Optical Astronomy Observatories, ${ }^{2}$ P.O. Box 26732, Tucson, Arizona 85726

\section{S. M. FABER}

UCO/Lick Observatories, Board of Studies in Astronomy and Astrophysics, University of California, Santa Cruz, Santa Cruz, California 95064

DOUGLas G. CURRIE

Department of Physics and Astronomy, University of Maryland, College Park, Maryland 20742

\section{S. P. EWALD}

Space Telescope Science Institute, 3700 San Martin Drive, Baltimore, Maryland 21218

EDWARD J. GROTH

Physics Department, Jadwin Hall, Princeton University, Princeton, New Jersey 08544

\section{J. JEFF HESTER}

Department of Physics and Astronomy, Arizona State University, Tempe, Arizona 85287

JON A. HoltzMAN

Lowell Observatory, 1400 Mars Hill Road, Flagstaff, Arizona 86001

\section{ROBERT M. LIGHT}

UCO/Lick Observatories, Board of Studies in Astronomy and Astrophysics, University of California, Santa Cruz, Santa Cruz, California 95064

EARL J. O'NEIL, JR.

Kitt Peak National Observatory, National Optical Astronomy Observatories, P.O. Box 26732, Tucson, Arizona 85726

EDWARD J. SHAYA

Department of Physics and Astronomy, University of Maryland, College Park, Maryland 20742

JAMES A. WESTPHAL

Division of Geological and Planetary Sciences, 170-25, California Institute of Technology, Pasadena, California 91125 Received 5 March 1992; revised 21 April 1992

\begin{abstract}
Analysis of $V$ band HST Planetary Camera images of the elliptical galaxy M32 shows that its nucleus is extremely dense and remains unresolved at even the HST diffraction limit. A combined approach of image deconvolution and model fitting is used to investigate the starlight distribution into limiting radii of $0.04(0.14 \mathrm{pc}$ at $700 \mathrm{kpc})$. The logarithmic slope of the brightness profile smoothly flattens from $\gamma=-1.2$ at $3.4 \mathrm{pc}$ to $\gamma=-0.5$ at $0.34 \mathrm{pc}$; interior to this radius the profile is equally consistent with a singular $\mu(r) \propto r^{-1 / 2}$ cusp or a small nonisothermal core with $r_{\mathrm{c}} \leqslant 0.37 \mathrm{pc}$. The isophotes maintain constant ellipticity into the center, and there is no evidence for a central point source, disk, dust, or any other substructures. The cusp model implies central mass densities $\rho_{0}>3 \times 10^{7} \mathscr{M}_{\odot} \mathrm{pc}^{-3}$ at the resolution limit and is consistent with a central $\mathscr{M}_{\bullet}=3 \times 10^{6} \mathscr{M}_{\odot}$ black hole; the core model implies $\rho_{0} \approx 4 \times 10^{6} \mathscr{M}_{\odot} \mathrm{pc}^{-3}$. From the viewpoint of long-term stability, we argue that a starlight cusp surrounding a central black hole is the more plausible interpretation of the observations. A core at the implied density and size without a black hole has a relaxation time of only $\sim 7 \times 10^{7} \mathrm{yr}$ and a short
\end{abstract}

\footnotetext{
'Based on observations with the NASA/ESA Hubble Space Telescope, obtained at the Space Telescope Science Institute, which is operated by AURA, Inc., under NASA Contract No. NAS 5-26555.

${ }^{2}$ The National Optical Astronomy Observatories are operated by the Association of Universities for Research in Astronomy, Inc., under cooperative agreement with the National Science Foundation.
} 
stellar collision timescale implying wholesale stellar merging over the age of the universe. The core would be strongly vulnerable to collapse and concomitant runaway stellar merging. Collapse may lead to formation of a massive black hole in any case if it cannot be reversed by formation of a binary from high-mass merger products. Regardless of the ultimate fate of the core, however, structural evolution of the core will always be accompanied by strong evolution of the core population-the constant isophote shape and absence of a central color gradient appear to show that such evolution has not occurred. In contrast, the high velocities around a black hole imply long relaxation and stellar collision times for the cusp population compared to the age of the universe.

\section{INTRODUCTION}

M32 appears to have a strong concentration of dark matter at its center based on the sharp central rise seen in its central velocity dispersion and rotation profiles (Tonry 1984; Tonry 1987; Dressler \& Richstone 1988). Tonry (1987) argues that the dark matter is in the form of a black hole with mass $\mathscr{M}_{\bullet}=3-10 \times 10^{6} \mathscr{M}_{\odot}$ largely from the rotation curve. Dressler \& Richstone (1988) infer $\mathscr{M}_{\bullet}=8$ $\times 10^{6} \mathscr{M}_{\odot}$, and further show that no model can be made to fit M32 with a constant central mass to light ratio. A more sophisticated reanalysis of the observations by Richstone et al. (1990) gives $\mathscr{M}_{\bullet}=0.7-3.0 \times 10^{6} \mathscr{M}_{\odot}$. Unfortunately, the black hole proposed for M32 strongly dominates the stellar dynamics only at radii below the seeing limit; even there the implied $\mathscr{M} / L$ never rises to extreme values when compared to typical $\mathscr{M} / L$ ratios for more luminous elliptical galaxies (Lauer 1985). Demonstrating that the dark matter is really in the form of a point mass, or that changes in the central stellar population cannot account for the central $\mathscr{M} / L$ increase, remain important problems. Goodman \& Lee (1989), for example, argued that a central cluster of dark stellar remnants could plausibly explain the M32 dynamics, a point demonstrated directly by Richstone et al. (1990).

Obtaining a clear picture of the M32 central star-light distribution is crucial to understanding its central mass distribution; M32 is thus a prime target for the Hubble Space Telescope. Tonry (1987) estimated the M32 core radius at $r_{\mathrm{c}}=0.27$ from observations taken in 1.0 full width at half-maximum (FWHM) seeing conditions. This core must be considered as only an upper limit; however, the photometry model adopted by Tonry (1987) does constrain the integrated luminosity in the inner arcsecond or so, and is sufficient to show that the mass-to-light ratio must increase towards the center. Lugger et al. (1992) obtained observations in conditions of only 0"40 FWHM, using the CFHT HRCAM. Interestingly, these observations yield the same core size deduced by Tonry (1987), even though the core is still unresolved, given the resolution criteria presented by Lauer (1985). The Lugger et al. (1992) observations are mainly useful in constraining changes in the central stellar population of M32. After matching their $R$ band observations to their slightly lower resolution $B$ band observations, Lugger et al. (1992) find $B-R$ to be constant to 0.01 mag from the very center out to $r=10^{\prime \prime}$, in agreement with Michard \& Nieto (1991) who show $U-R$ constant to $0.05 \mathrm{mag}$ for $r<10^{\prime \prime}$. Lugger et al. (1992) also find no evidence for any special central components such as a disk, star cluster, or dust that might complicate interpretation of the light profile. Furthermore, they find the isophotes to maintain constant shape into the center with no deviations from ellipticity larger than $0.2 \%$.

The HST observations presented here go beyond the resolving power of the best ground-based observations, showing that the M32 nucleus is extremely dense, with central mass density $\rho_{0}>4 \times 10^{6} \mathscr{M}_{\odot} \mathrm{pc}^{-3}$; the nucleus, in fact, remains unresolved at even the HST diffraction limit. The star-light distribution is equally consistent with a stellar cusp surrounding a $\mathscr{M}_{\bullet}=2.8 \times 10^{6} \mathscr{M}_{\odot}$ black hole, or a small nonisothermal core; however, if M32 does not have a central black hole, then its core is vulnerable to rapid structural evolution. The simulations of Quinlan \& Shapiro (1990) show that one possible outcome of core collapse of systems similar to the M32 nucleus is formation of a black hole in any case. M32 thus appears to be an excellent site to investigate formation of a black hole by collapse of a stellar system.

\section{OBSERVATIONS AND ANALYSIS}

\subsection{Observations and Basic Reduction}

$H S T$ observations of M32 were obtained on day 229 of 1991 with the PC or high resolution mode of the Wide Field/Planetary Camera (WFPC). Briefly, the PC comprises four CCD cameras imaging a contiguous $66^{\prime \prime} \times 66^{\prime \prime}$ field of view. Each CCD has an $800 \times 800$ pixel format with 0 ".044 per pixel. Further description of the camera is presented by Griffiths (1989). One $20 \mathrm{~s}$ and four $100 \mathrm{~s}$ exposures were obtained with filter F555W, which corresponds roughly to the Johnson $V$ band. We chose not to obtain images in additional colors since, as noted above, groundbased imaging at subarcsecond resolution showed $B-R$ to be constant in the center to $0.01 \mathrm{mag}$ (Michard \& Nieto 1991; Lugger et al. 1992), and spherical aberration blurring greatly compromises detection of subtle color gradients in the inner arcsecond of the galaxy.

The signal level at the galaxy center in the $100 \mathrm{~s}$ images was $2.0 \times 10^{4} \mathrm{e}^{-}$per pixel; however, the signal to noise ratio is actually limited by the finite number of stars per pixel. Tonry \& Schneider (1988) show that the transition from photon to "luminosity fluctuation" dominance of variance in the surface brightness occurs when the "typical star," which for populations typical of elliptical galaxies has $M_{V} \approx 0.4$, contributes more than 1 photon per exposure. For a distance to M32 of $700 \mathrm{kpc}$, this luminosity corresponds to $m_{V}=24.6$, or $\sim 190$ photons in a $100 \mathrm{~s} \mathrm{PC}$ 
exposure; we are thus strongly dominated by star rather than photon statistics.

The telescope was guided in coarse track during the exposure sequence. The second $100 \mathrm{~s}$ exposure suffered from an unacceptable amount of guiding jitter and was discarded. The jitter was $0.039 \mathrm{rms}$ in the remaining exposures, which is significant but acceptable. The nucleus of M32 was positioned in the center of CCD PC 6, 9.2 from the nearest edge of the field; for the present analysis only the data from PC 6 were used. The position of the nucleus agrees to the milliarcsecond level in the last two exposures but shifted by $4 \times 10^{-3}$ arcsec from the first exposure; after removal of cosmic rays all three images were shifted to a common center.

An accurate PSF is crucial to the analysis of M32, given the severe spherical aberration present in the HST primary (Burrows et al. 1991). A fast exposure of a bright SAO star was obtained shortly after the M32 exposures were complete; unfortunately the exposure level was unacceptably low and the star was positioned 102 pixel away from the M32 center, a distance over which significant changes in the PSF halo structure can occur. We decided instead to use a composite F555W PSF built from four exposures obtained six weeks earlier, all of which had high signal levels and were positioned about 40 pixel closer to the M32 center. The halo of the composite PSF matched the structure of the SAO star observed after M32 to within the photon noise, so it did appear that the optics of the telescope were stable over the six weeks. A separate concern is the method of interpolation used to register the individual PSF exposures. Because the PSF core is undersampled at F555W, we used third-order Lagrangian rather than sincfunction interpolation; the latter method would introduce strong "ringing" artifacts if used on undersampled data. While there may be small errors in the representation of the PSF core thus derived, we argue that the shape of the PSF core is not itself critical to analysis of the M32 central structure, but rather the ratio of core to halo light. We were concerned with the amount of guiding jitter, so we obtained telemetry of the detailed spacecraft motion and constructed a jitter PSF that was convolved with the composite sharp PSF The major effect was significant smearing of the sharp core, but, as we discuss below, use of the jittered versus unjittered PSF makes only subtle changes in the measured brightness profiles. Since the introduction of jitter made large changes in the PSF core, such results show that subtle errors in representation of the PSF core shape are not important. As discussed below, M32 does not have a point source nucleus and has very little "power" at the diffraction limit of the telescope. This also means that shifting the individuals images of M32 to a common center introduces no significant errors.

At the time of the observations, calibration of the WFPC was still in a preliminary state; however, all reduction steps outlined by Lauer (1989) could be completed. The flatfield for F555W was generated from several "streak" frames and gave generally excellent results. A number of compact defects remained in the image, however, due to material on the CCD field flatteners. The de-

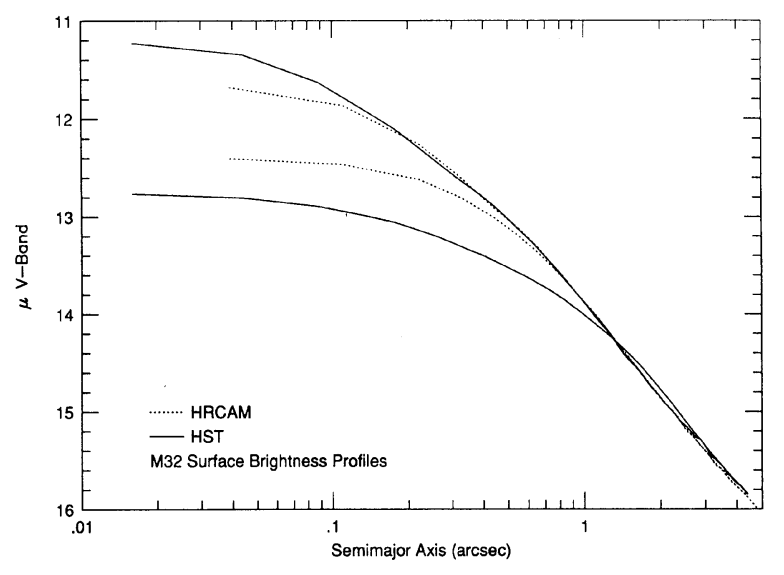

FIG. 4. Observed and deconvolved brightness profiles of M32 as observed with HST (solid lines), and the CFHT HRCAM (dotted line) profiles of Lugger et al. (1992). The deconvolved profiles are the upper two curves in the figure. Note the excellent agreement of the deconvolved profiles for $r>0$. 2 . In this figure and all that follow the central point in the profiles is assigned a radius of 0.016 , which is the pixel size of 0 " 044 divided by $2 \sqrt{2}$.

fects closest to the M32 nucleus were clipped out and replaced with a model reconstructed from the surface photometry profiles. Cosmic rays were identified by intercomparison of the three $100 \mathrm{~s}$ exposures; the analysis below was performed on the sum of the three $100 \mathrm{~s}$ exposures cleaned of cosmic rays (the $20 \mathrm{~s}$ exposure was not used in this analysis).

The central portion of the reduced image is presented in Fig. 1 [Plate 78]. The same image after 160 iterations of Lucy-Richardson deconvolution (Lucy 1974; Richardson 1972) is presented in Fig. 2 [Plate 79]. Figure 3 [Plate 80] shows the deconvolved image divided by a model image reconstructed from the deconvolved surface brightness profile (the model assumes that M32 can be described completely by the set of elliptical isophotes measured by the profile). M32 is clearly extremely concentrated in the center; however, we find no morphological peculiarities such as dust lanes, disky or boxy isophotes. We also find no evidence of nonconcentric isophotes over the inner few arcseconds of M32 to a limit of 0.012, or a 0.041 pc change in isophote center. Further, there is no evidence of a central nuclear point source. As shown below, the deconvolved profile is smooth, showing no sign of an inflection that would be expected if a strong point source were present. We set limits on any point source present at $m_{V} \geqslant 19.5$, or $L_{V} \leqslant 6.7 \times 10^{3} L_{\odot}$. This limit comes from the assumption that the slight increase in brightness of the centralmost point over the next point out in the deconvolved profile (Fig. 4) might be due to a point source. The limit quoted reflects the amount of light that must be removed to suppress the increase in brightness of the central point as estimated by subtracting the deconvolved PSF (that is the original PSF itself processed by Lucy deconvolution) from the deconvolved image.

The most striking feature of the deconvolved image is its prickly appearance due to the luminosity fluctuations 
TABLE 1. M32 deconvolved surface-brightness profile.

\begin{tabular}{|c|c|c|c|c|c|c|c|c|c|c|c|}
\hline $\mathrm{R}$ & $\mu_{V}$ & PA & $\epsilon$ & $\mathrm{R}$ & $\mu_{V}$ & PA & $\epsilon$ & $\mathrm{R}$ & $\mu_{V}$ & PA & $\epsilon$ \\
\hline 016 & .229 & 169.0 & 0.152 & 496 & & 161.0 & & 2.992 & & & \\
\hline 0.044 & & 9.0 & & 340 & & 161.8 & & 036 & & & \\
\hline 0.088 & & 169.0 & 0.152 & 1.584 & & 161.8 & & 3.080 & & 160.2 & 0.266 \\
\hline 0.132 & 5 & 169.0 & 0.191 & 1.628 & & 161.8 & & 3.124 & & 160.7 & 0.256 \\
\hline 176 & & 174.0 & 0.256 & 1.672 & 14.6 & 161.8 & & 3.168 & 15.457 & 161.0 & 0.256 \\
\hline 0.220 & & 168.6 & & 1.716 & & 160.9 & & 3.212 & & 161.0 & 0.256 \\
\hline 0.264 & 12.455 & 164.5 & 0.256 & 1.760 & & 160.8 & 0.282 & 3.256 & & 161.0 & 0.262 \\
\hline 0.308 & 2.5 & 163.2 & 0.259 & 1.804 & 14.7 & 160.8 & 82 & 3.300 & & 161.0 & 0.262 \\
\hline 0.352 & & 163.2 & & 1.848 & & 160.8 & & 3.344 & & 161.0 & 0.262 \\
\hline 0.396 & 1 & 163.2 & & 1.892 & & 160.9 & & & & 161.0 & 268 \\
\hline 0.440 & 12.891 & 163.1 & & 1.936 & 14. & 161.2 & 0.291 & 3.432 & & 161.0 & 0.268 \\
\hline 0.484 & 1 & 163.1 & & 1.980 & 14. & 161.2 & & 3.476 & & 161.5 & 0.268 \\
\hline 528 & & 163.1 & & 2.024 & & 161.2 & & 3.520 & & 162.1 & 268 \\
\hline 0.572 & 8 & 163.2 & & 2.068 & & 161.2 & & 3.564 & & 162.1 & 0.268 \\
\hline 0.616 & 13.2 & 163.3 & 0.262 & 2.112 & 14. & 161.2 & 0.269 & 3.608 & & 162.1 & 0.248 \\
\hline & & 163.3 & & 2.156 & & 161.2 & & & & 162.1 & 248 \\
\hline 0.704 & & 163.3 & & 2.200 & & 161.2 & & 3.696 & & 159.8 & 0.248 \\
\hline 748 & 1 & 164.0 & & 2.244 & 15. & 161.2 & & 3.740 & & 159.5 & 0.252 \\
\hline 0.792 & & 164.0 & & 2.288 & & 162.6 & 267 & 3.784 & & 159.5 & 0.257 \\
\hline 0.836 & & 164.0 & & 2.332 & & 163.3 & & & & 159.5 & 0.257 \\
\hline 0.880 & & 164.0 & & 2.376 & & 163.3 & & 3.8 & & 159.5 & 0.257 \\
\hline 0.924 & 13.78 & 162.5 & & 2.420 & 15. & 163.3 & & 3.916 & & 160.6 & 0.252 \\
\hline 0.968 & 13.8 & 162.0 & 272 & 2.464 & 15. & 163.3 & 0.282 & 3.960 & & 161.7 & 0.252 \\
\hline 1.012 & & 161.0 & & 2.508 & & 162.0 & & & & 162.0 & 0.252 \\
\hline 1.056 & 13.967 & 161.0 & & 2.552 & 15.168 & 160.7 & & 4.048 & & 162.2 & 0.258 \\
\hline 1.100 & 14.025 & 161.0 & 0.276 & 2.596 & 15.190 & 159.7 & 0.283 & 4.092 & 15.752 & 162.2 & 0.263 \\
\hline 1.144 & 14.080 & 161.0 & & 2.640 & 15.211 & 158.7 & & 4.136 & 15.754 & 162.2 & 0.273 \\
\hline 1.188 & 14.129 & 161.4 & & 2.684 & & 158.7 & & 4.180 & & 162.2 & 0.274 \\
\hline 1.232 & 14.177 & 161.4 & 0.275 & 2.728 & 15.256 & 158.7 & 0.281 & 4.224 & 15.791 & 161.4 & 0.275 \\
\hline 1.276 & 14.227 & 161.4 & 0.272 & 2.772 & 15.273 & 158.7 & 0.285 & 4.268 & 15.803 & 160.5 & 0.279 \\
\hline 1.320 & 14.281 & 161.4 & & 2.816 & & 158.7 & & 4.312 & & 160.2 & 0.281 \\
\hline 1.364 & 14.339 & 161.0 & 0.263 & 2.860 & 15.286 & 159.1 & 0.304 & 4.356 & 15.826 & 160.2 & 0.287 \\
\hline 1.408 & 14.387 & 161.0 & 0.263 & 2.904 & 15.303 & 159.4 & 0.304 & 4.400 & 15.837 & 160.4 & 0.290 \\
\hline 1.452 & 14.432 & 161.0 & 0.263 & 2.948 & 15.324 & 159.5 & 0.304 & ... & $\ldots$ & $\ldots$ & $\ldots$ \\
\hline
\end{tabular}

noted above. As discussed below, the central surface brightness exceeds $\mu_{V}=11.5$, which corresponds to $\approx 400$ typical stars per pixel. The resolution is somewhat poorer than a pixel in even the deconvolved image, but clearly the rms surface brightness fluctuation will be close to 0.05 mag, which are the measured residuals in Fig. 3.

\subsection{Measurement of the M32 Starlight Profile}

The brightness profiles of M32 before and after deconvolution are presented in Fig. 4 and the deconvolved profile is presented in Table 1. Isophote ellipticity, $\epsilon=(1-b / a)$, is shown in Fig. 5. The ellipse fitting software of Kent (1983) as modified by Lauer (1985) for high-resolution applications was used. Photometric calibration was provided by published $V$-band aperture photometry (Burstein 1989).

The center of M32 is compact and deconvolution of the spherical aberration blurring produces a substantial correction. Taken at face value, the deconvolved profile shows that for $r<1$ " the steep gradient in the profile seen at larger radii begins to flatten out and may even vanish as $r \rightarrow 0$. As we will show below, the decrease in the logarithmic slope of the profile is real, but any true core remains unresolved at the limiting resolution of $H S T$. We will make this case be testing the deconvolution algorithm on models and by fitting models blurred by spherical aberration directly to the data. 


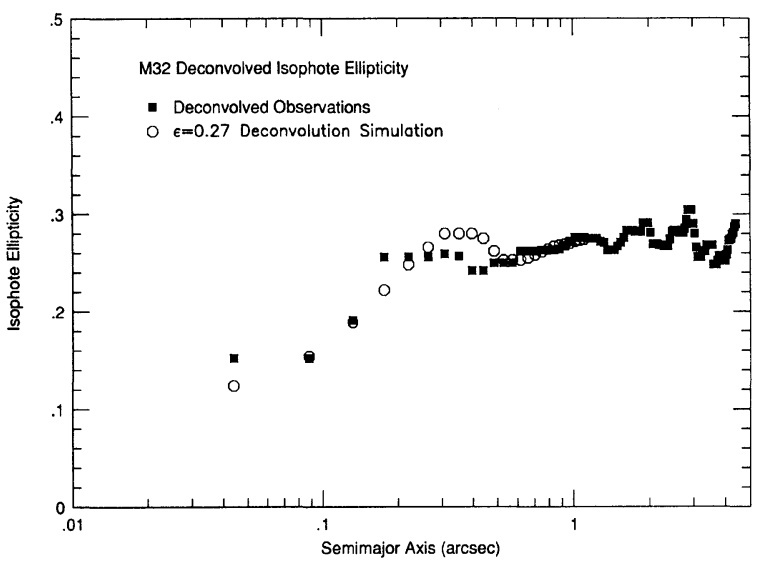

FIG. 5. Deconvolved isophote ellipticity profile. Solid symbols show the ellipticity as measured from the deconvolved image. Open symbols show measurements from a deconvolution simulation that assumed constant $\epsilon=0.27$ as $r \rightarrow 0$.

An important point about deconvolution is that complete recovery of the unblurred object can never be achieved; the process effects a partial removal of the blurring, but the deconvolved profile still has limited, albeit improved resolution. In the case of Lucy-Richardson deconvolution, the resolution gain is controlled by the number of iterative deconvolution cycles. While this apparently arbitrary nature of Lucy-Richardson is disturbing to some, it must be understood that the real limiting factor in deconvolution is the signal-to-noise ratio, a factor that controls either implicitly or explicitly the sharpening power of all other deconvolution algorithms as well.

Another important point is that the effect of any deconvolution algorithm can be estimated by simulation, which we do now for M32. As we discuss in the next section, the best fitting model for M32 appears to include a central cusp

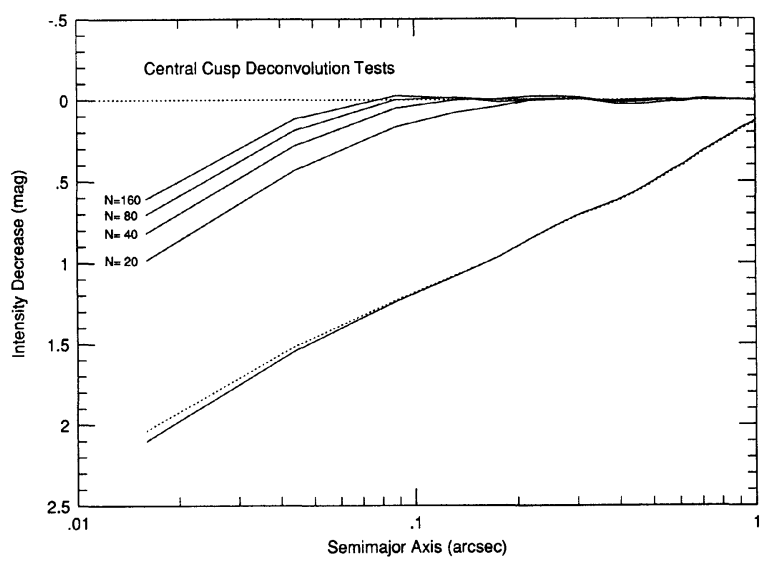

FIG. 6. Simulated deconvolved profiles compared to a model with an intrinsic $\gamma=-1 / 2$ cusp for $r<0$ ". 15 . The bottom solid trace shows the decrease in central intensity of the model after convolution with the HST PSF. The dotted line shows the effect of neglecting FGS jitter; its central intensity is 0.06 mag brighter. The upper set of solid traces are the model-deconvolved profile residuals after 20, 40, 80, and 160 iterations of Lucy-Richardson deconvolution. that consists of a power law with $\gamma \approx-1 / 2$ for $r<0$ ". 15 that smoothly steepens to $\gamma \approx-1.3$ at large radii. Deconvolution simulations on images generated from this model [see Eq. (1)], are presented in Fig. 6, which shows differences between the intrinsic unblurred model and brightness profiles extracted at various stages in the deconvolution process. Constant isophote ellipticity and position angle appropriate to the M32 envelope has been assumed; we have also assumed that photon statistics are the only noise source, which means that the simulations may be somewhat optimistic on how much of the centralmost structure can be recovered. The lowest trace in Fig. 6 shows the full effect of spherical aberration-the central intensity decreases by over 2 mag, and blurring effects extend to beyond 1". We have included the FGS jitter blurring in these simulations, although we show a full convolution without jitter in Fig. 6 as well to show that jitter makes only a small difference on top of spherical aberration-this bolsters our earlier claim that small errors in estimating the PSF core are of no consequence. The upper set of four traces in Fig. 6 shows the effects of increasing the number of Richardson-Lucy iterations, beginning with 20 iterations and increasing to our present limit of 160 iterations in powers of two. As few as 20 iterations are sufficient to remove most of the blurring and to recover the brightness profile to $0.01 \mathrm{mag}$ for $r>0$ ". 2 . By 80 iterations we have recovered the profile to the 0.01 mag level for $r>0$ " 1 ; the effect of the next 80 iterations is to boost the central two points by about 0.1 mag. We conclude that the deconvolved profile can be used directly for $r \geqslant 0$ ". 1 , but residual blurring exists for smaller radii, from the simulations, the final resolution is estimated to be about 0"07 FWHM.

One question might be whether one could use the simulations presented in Fig. 6 to make a correction to account for the residual blurring effects. Unfortunately, such corrections depend on the true nature of the intrinsic profile at $r<0$ ". 1 . What we are seeing here is not the effect of the spherical aberration halo but the sharp cutoff on spatial information caused by the diffraction core of the PSF itself. One might push the deconvolution harder, but a better approach is to use the deconvolved profile for $r>0$ ". 1 to motivate plausible models for the structure at $r<0$ ". 1 - this works because we are strongly constraining the possible outcome rather than continuing ever onward with a fully general deconvolution algorithm. This will be done in the next two sections, where we fit models to the M32 image prior to deconvolution to show the range of intrinsic profiles compatible with the observations.

Before discussing the brightness profile further, we present in Fig. 5 a simulation of the effects of deconvolution on isophote ellipticity. The deconvolved profile appears to have constant position angle of $161^{\circ}$ and constant ellipticity $\epsilon=0.27$ for $r>1^{\prime \prime}$. The deconvolution simulations presented above assumed constant isophote shape into the center. The apparent decrease in ellipticity seen in Fig. 5 is well matched by the deconvolution simulation, showing that it is due only to limitations in the deconvolution. M32 appears to have constant ellipticity into its center. 
The best check of the present deconvolved profile would be direct observation of M32 without the strong effects of spherical aberration. While $H S T$ ultimately offers the best resolution for galaxy profiles, the effects of spherical aberration actually extend to larger radii than are affected by seeing in the best ground-based images. In Fig. 4 we compare our profile with the HRCAM $R$-band profile of Lugger et al. (1992) taken in 0.4 FWHM seeing. The HRCAM and HST profiles match very poorly at small radii prior to deconvolution-indeed the HRCAM data actually record a higher raw surface brightness. After deconvolution, however, both profiles agree to $0.01 \mathrm{mag}$ for $r>0$ " 4 and to $0.03 \mathrm{mag}$ for $r>0$." 2 ; given the totally different character of these two datasets, we consider their excellent agreement after deconvolution a striking validation of Lucy deconvolution. This result also shows that $V-R$ is constant for $r>0$ " 2 . We now see that for $r<0$ ". $2 H S T$ is providing data of superior resolution; the HRCAM deconvolved profile falls below the $H S T$ data by $0.45 \mathrm{mag}$ at the center.

\subsection{Central Cusp Models}

The hypothesis that we will now test is whether or not M32 has a cusp or a core at its center. By "core" we mean a region interior to which the surface brightness profile levels off and becomes constant as $r \rightarrow 0$, implying a more or less constant central stellar density, even if the core shape is not precisely isothermal; by "cusp" we mean a central brightness profile that maintains a significant logarithmic slope in the center, so that the central surface brightness would be formally singular. The apparent flattening of the profile at $r<0$ ". 1 is clearly close to our resolution limit, and the experiments above argue that it may be merely an artifact of residual blurring in the deconvolved profile. The true profile might be one that has a steep logarithmic slope at large $r$, transitioning smoothly to a shallower cusp at small $r$.

After some experimentation, we decided to try a model of the form

$$
I(r)=I_{0} r^{\alpha}\left[1+(r / a)^{2}\right]^{\beta},
$$

assuming constant P.A. $=161^{\circ}$ and $\epsilon=0.27$ in the center. As $r \rightarrow 0$ we have $I(r) \propto r^{\alpha}$, and as $r \rightarrow \infty$ we have $I(r) \propto r^{\alpha+2 \beta}$. A least-squares fit of the model convolved with the HST PSF to the image data directly gives $\mu_{0} \equiv 2.5 \log \left(I_{0}\right)=13.04$ ( $V$ band ), $a=0$ ".378, $\alpha=-0.531$, and $\beta=-0.375$.

This model is plotted in Fig. 7 against the deconvoluted profile, and its residuals with respect to the data as observed prior to deconvolution are shown in Fig. 8. The match is excellent, showing that M32 can possess a central cusp, with $\gamma \approx-1 / 2$ for $r<0.6$ pc. As a check that the profile slope really does decrease to this value, we show fits in Fig. 8 assuming inward extrapolation of $\gamma=-1$ and $-3 / 4$ power laws from radii of 0.47 and 0 ." 22 , which are the points at which the model above reaches these slopes locally. Both models predict too much light at the center, showing that as $r \rightarrow 0, \gamma$ is much closer to $-1 / 2$ than

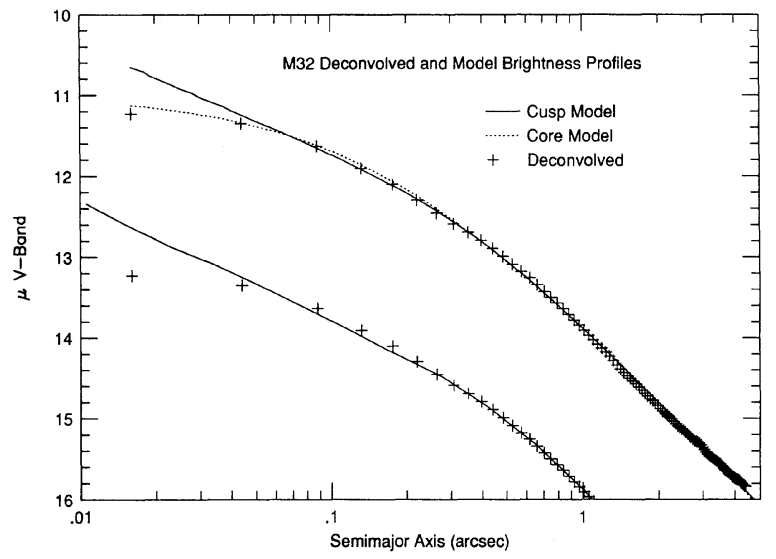

FIG. 7. Comparison of the M32 deconvolved brightness profile (crosses) to models of the central light distribution. The upper solid line is the best fitting model with a central cusp, and the dotted line is the best-fitting model with a central core. The divergence at the center of the two models is not significant. The deconvolved profile still has limited resolution, and falls below both models which have not been convolved with any PSF in this figure. The lower trace shows the deconvolved profile offset by 2 mag matched with the Young (1980) 3.0 black hole cusp model.

$-3 / 4$. Finally, we again mention the effects of jitter. $\mathrm{Ne}-$ glecting jitter underestimates the amount of PSF blurring, leading in turn to a $\sim 20 \%$ decrease in the $\gamma$ estimated. Uncertainties in the central surface brightness of the M32 image itself leads to a $10 \%$ uncertainty in $\gamma$, so our best estimate including jitter would be $\gamma=-0.53 \pm 0.05$ for $r$ $<0$ ". 10 .

\subsection{Central Core Models}

The central decrease in the deconvolved brightness profile slope might suggest that M32 really does have a small core. We tested this second hypothesis by fitting models of the form

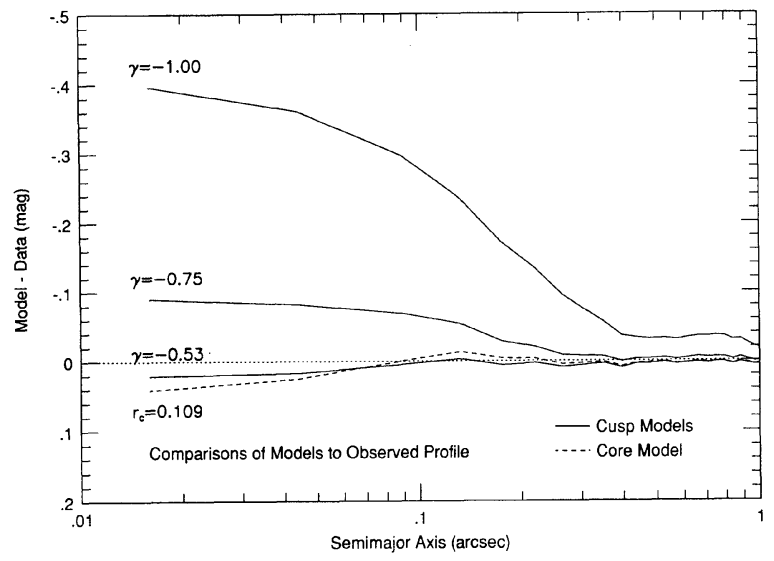

FIG. 8. Residuals of brightness distribution after convolution compared to the brightness profile of the M32 image as observed prior to deconvolution. The solid lines show the sequence of cusp models with $\gamma=-1,-3 / 4$, and the best fitting $\gamma=-0.531$. The dashed line shows the best-fitting core model, which implies $r_{\mathrm{c}}=0$ ". 109 


$$
I(r)=I_{0}(1+r / a)^{\beta} \text {. }
$$

Note that the $r / a$ term is now raised only to a single power; since this model ultimately has a greater change in slope between its inner and outer regions than in the cusp model, the transition as controlled by the term in the denominator must be more gradual. The core is thus strongly nonisothermal, since the transition between envelope and core is much more gradual than in an isothermal core. Further, the linear derivative does not go to zero at the origin as it does in an isothermal core. This implies a singular central density, although it clearly would be easy to force the slope to zero at small radii with little effect on the quality of the fit. As shown below, the stellar density implied by this model increases very slowly interior to the core, and the effective central density can be estimated with little error from the central surface brightness and core radius from the formula appropriate to an isothermal core.

The best fit model has $\mu_{0}=10.99, a=0$ ". 166 , and $\beta=$ -1.37 ; the implied core radius, or half-power point, is $r_{\mathrm{c}}=0.11$ or $0.37 \mathrm{pc}$. This fit compared to the deconvolved profile is shown in Fig. 7 and its residuals with respect to the data is observed are shown in Fig. 8. This fit is plausible given the uncertainties in the data, but it matches the data slightly less well than the cusp model, underestimating the central flux while being too bright at $r \approx 0$ ". 1 . The problem is that, even in the center, the true decrease in slope is still more gradual than that incorporated into this already gradual model. We regard this core radius as only an upper limit rather than a true detection of a core, given that its size is only slightly larger than the HST diffraction limit and the demonstration that the data are fit equally well by the cusp model.

We emphasize that both the cusp and core models describe the same behavior for $r>0$ " 1 , the region of the profile that we consider to be well determined, and show a large intensity difference only at the central point in the profile. Figure 9 shows the local logarithmic slope of both models as a function of radius; both models have the same slope for $r>0$ ". 1 , despite their differences at smaller radii.

\section{DISCUSSION}

Objective analysis of the central M32 starlight distribution shows that it is equally consistent with an $r^{-1 / 2}$ singular cusp or a small, $r_{\mathrm{c}}=0.37 \mathrm{pc}$ core. We now ask first, what are the implications either way for the existence of a central black hole, and second, what physical arguments might lead us to prefer one model over the other. A starlight cusp gives direct support to the existence of a massive black hole at the center of M32 (Young 1980). A small core may be consistent with a hole, but it also raises the possibility that the core is more analogous to a dynamically evolving star cluster, with dark matter in the form of stellar-mass remnants rather than a large black hole. Both pictures will be examined in turn.

Even with a core, the M32 central mass density is extremely high. Figure 10 shows the mass density profiles obtained by Abel inversion of the cusp and core models

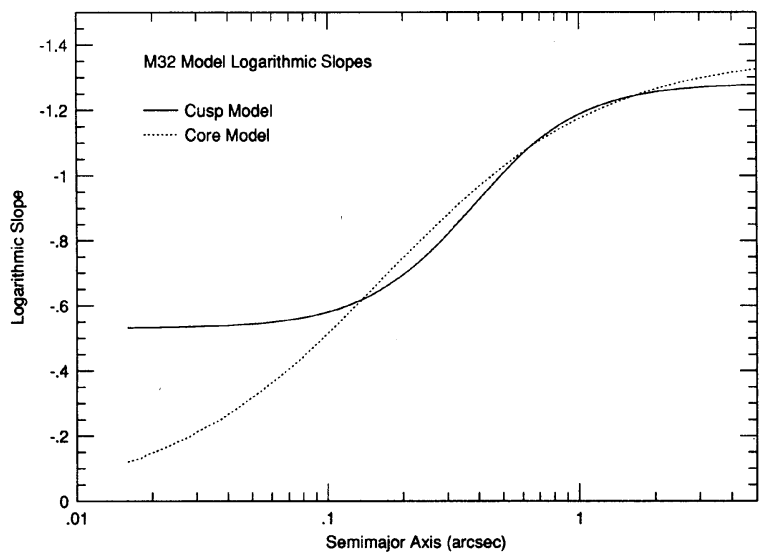

FIG. 9. Logarithmic slopes of the best-fitting cusp model (solid) and core model (dotted). Note the excellent agreement of the models for $r>0$. 1 .

inferred from the starlight alone; a $V$-band $\mathrm{M} / \mathrm{L}=2.0$ has been assumed, which is equivalent to the $B$-band $\mathrm{M} / \mathrm{L}$ $=2.65$ derived for the star light by Tonry (1987). Tonry's model, which has mass density $\rho(r)=9.7 \times 10^{5} \mathscr{M}_{\odot} / \mathrm{pc}^{3}$ $\times\left[1+(r / 0.61 \mathrm{pc})^{2}\right]^{-1.1}$, is also shown for comparison (this includes a $4 \%$ change in Tonry's zero point); the present results clearly show that M32 is significantly denser in the center than was inferred from ground-based observations. As noted in Sec. 2.4, the core model formally has an infinite central density; however, we estimate an effective central density at $\rho_{0}=4.0 \times 10^{6} \mathscr{M}_{\odot} \mathrm{pc}^{-3}$ from the relation $\rho_{0}=I_{0} / 2 r_{\mathrm{c}}$ (Peterson \& King 1975). The cusp reaches densities $\rho>3 \times 10^{7} \mathscr{M}_{\odot} \mathrm{pc}^{-3}$ at the central pixel of our observations, almost an order of magnitude higher. Despite these high central densities, the integrated mass profiles (Fig. 11) imply only a modest increase in the total stellar mass within the central $1 \mathrm{pc}$. It remains true that there is not enough mass in stars within the central $1 \mathrm{pc}$ to be the sole source of the sharp rotation and dispersion

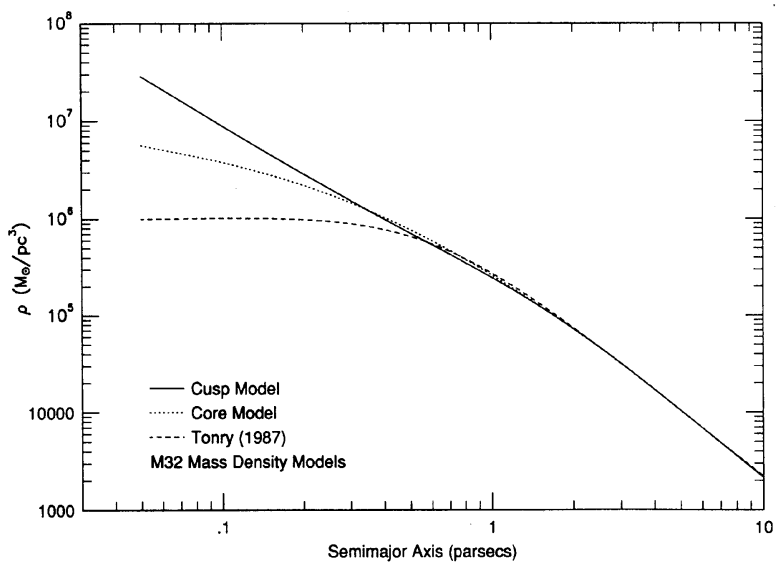

FIG. 10. Mass density profiles for the cusp model (solid), core model (dotted), and the Tonry (1987) core model (dashed). The Tonry model has been adjusted upwards by $4 \%$ to match the present results at large radii. 


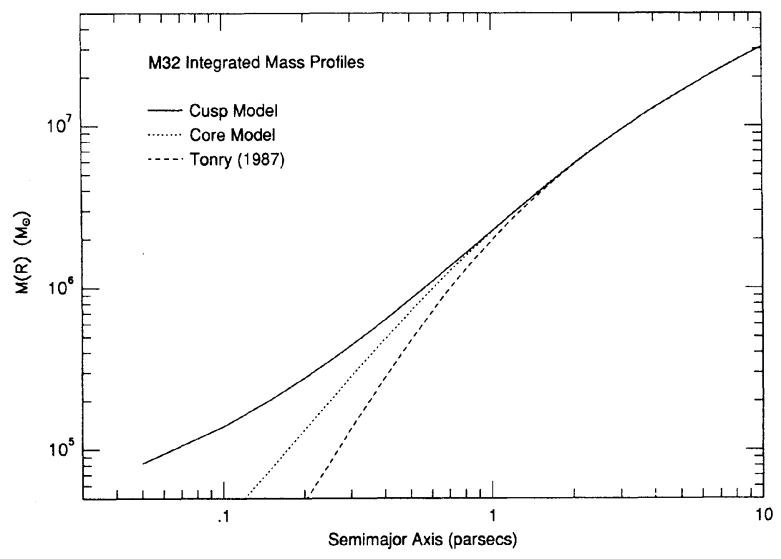

FIG. 11. Integrated mass profiles for the cusp model (solid), core model (dotted), and the Tonry (1987) core model (dashed). The Tonry model has been adjusted upward by $4 \%$ to match the present results at large radii.

profiles; however, the more concentrated light is clearly probing a region closer to the center than was assumed by Tonry, so a reanalysis of existing dynamical data with the new profiles would probably yield a modest reduction in the black hole mass inferred.

As Goodman \& Lee (1989) have emphasized, at high mass densities there are two processes that may lead to evolution of the M32 central structure. Two-body interactions between stars in the core induce relaxation and, ultimately, core collapse. The relaxation timescale is

$$
t_{\mathrm{r}}=\frac{0.34 \sigma^{3}}{G^{2} \mathscr{M} \rho \ln (0.4 N)},
$$

where $\sigma$ is the one-dimensional velocity dispersion, $\mathscr{M}$ is the typical stellar mass, $\rho$ is the total mass density, and $N$ is the number of stars in the system (Binney \& Tremaine 1987). On a longer timescale, the stars may actually collide, leading to the formation of tidal binaries, stellar mergers, or disruption. The collision timescale for any star is

$$
t_{\mathrm{c}}=\left[16 \sqrt{\pi n \sigma r_{p}^{2}}\left(1+\frac{G \mathscr{M}}{2 \sigma^{2} r_{p}}\right)\right]^{-1},
$$

where $n$ is the stellar number density, and $r_{p}$ is the stellar radius (Binney \& Tremaine 1987). We will show that in a cusp model where the black hole mass dominates, both relaxation and collisions are only marginally important over the age of the universe, while in a core model without a black hole and consequential high central velocity dispersion, dynamical evolution due to both processes is rapid.

\subsection{The Star-light Cusp Model}

A central star-light distribution in the form of a singular $r^{-1 / 2}$ power law suggests direct photometric evidence of the black hole inferred from the M32 spectroscopic observations. Young (1980) shows how the central structure of a galaxy evolves as a central black hole grows adiabatically from an initially isothermal core. Close to the hole, the projected density of stars follows an $r^{-1 / 2}$ cusp, precisely the slope of the cusp model found above. The form of the profile at larger radii depends on the ratio of the hole mass to that in the preexisting core. In the case of M32, the power-law slope becomes steeper at larger radii, suggesting that the black hole must dominate any previously existing core. The best-fitting Young (1980) model has a black hole to core mass ratio $\mathscr{M} / 4 \pi \rho_{0}\left(r_{\mathrm{c}} / 3\right)^{3}=3 \pm 1$, and is shown in Fig. 7 compared to the deconvolved profile-as expected, it appears to be indistinguishable from the ad hoc cusp model discussed in Sec. 2.3. Choice of this model implies an original core with $r_{\mathrm{c}}=3.0 \mathrm{pc}$ and $\rho_{0}=8.4 \times 10^{4}$ $\mathscr{M}_{\odot} \mathrm{pc}^{-3}$, assuming a global $\mathbf{M} / \mathbf{L}=2.0$; the implied black hole mass is $\mathscr{M}_{\bullet}=(2.8 \pm 0.9) \times 10^{6} \mathscr{M}_{\odot}$.

This black hole mass is somewhat low compared to the 3-10 $\times 10^{6} \mathscr{M}_{\odot}$ range estimated by Tonry (1987) and the $8 \times 10^{6} \mathscr{M}_{\odot}$ estimated by Dressler $\&$ Richstone (1988) but is at the high end of the $0.7-3.0 \times 10^{6} \mathscr{M}_{\odot}$ range estimated more recently by Richstone et al. (1990). This last result is intriguing but is derived from models that assume spherical symmetry and no rotation, neither of which is correct for M32; although the Lee \& Goodman (1989) models that consider growth of a black hole in a rotating system appear similar to the Young (1980) models. Furthermore, the present model is at the high end of the black hole masses modeled by Young (1980), and it is not clear how accurately the black hole mass can be deduced from this procedure once its mass strongly dominates the core, even within the framework of the model assumptions. The real proof of a black hole must come from spectroscopic observations at matching resolution.

Traditionally, the approach to modeling the photometry and dynamics of galactic nuclei has been conservative, seeking to explain the observations without recourse to a black hole. In terms of stability, however, the more conservative approach may actually be to accept a central black hole. The high velocity dispersion near a hole leads to long timescales for both relaxation and stellar collisions. Within the central $0.5 \mathrm{pc}$ for M32, the stellar mass density of the cusp model is

$$
\rho(r)=2.8 \times 10^{5}\left(\frac{1 \mathrm{pc}}{r}\right)^{3 / 2} \mathscr{M}_{\odot} \mathrm{pc}^{-3}
$$

The dispersion within the cusp will be dominated by the black hole, which gives

$$
\sigma(r)=93\left(\frac{1 \mathrm{pc}}{r}\right)^{1 / 2}\left(\frac{\mathscr{M}_{\bullet}}{3 \times 10^{6} \mathscr{M}_{\odot}}\right)^{1 / 2} \mathrm{~km} / \mathrm{s} .
$$

From Eq. (3), this implies $t_{\mathrm{r}}=4 \times 10^{9} \mathrm{yr}$, independent of radius. While this result is less than the age of the universe, it takes a few relaxation timescales for significant structural evolution to occur, and it is much longer than the relaxation time for the core model calculated in the next section.

The timescale for physical collisions between stars decreases towards the center. Close to the black hole, where $\sigma \gg \sqrt{G \mathscr{M} / 2 r_{p}}$ 


$$
t_{\mathrm{c}}=2.6 \times 10^{12}\left(\frac{r}{1 \mathrm{pc}}\right)^{2}\left(\frac{3 \times 10^{6} \mathscr{M}_{\odot}}{\mathscr{M}_{\bullet}}\right)^{1 / 2} \mathrm{yr},
$$

where we have assumed solar radii for all stars. Equation (7) is valid for $r<0.09 \mathrm{pc}$, or $t_{\mathrm{c}}<2 \times 10^{10} \mathrm{yr}$; at larger radii, $t_{\mathrm{c}} \propto r$, rather than $r^{2}$. Stellar collisions will be significant within the cusp, but will only affect the structure of the cusp itself at radii well below our resolution limits. In passing, we note that, while the density profile is formally singular, it does not imply an absurd central number density of stars. Even assuming that Eq. (5) is valid all the way into the center, one must integrate out to $r=12 \mathrm{AU}$ to enclose one solar mass.

The one serious difficulty for the black hole model is what Goodman \& Lee (1989) refer to as the "luminosity problem," or the apparent lack of any nuclear activity in M32 at all (see Tonry 1987 for a review). Although the timescale for relaxation to affect the structure of the cusp is probably longer than a Hubble time, individual stars will diffuse into the black hole loss cone at a significant rate. For black hole cusps similar to that proposed here, Murphy et al. (1989) calculate a central mass infall rate (that also has a significant stellar mass-loss component) of $10^{-4}-10^{-5} \mathscr{M}_{\odot} \mathrm{yr}^{-1}$, which, with an assumed efficiency of $\eta \approx 0.1$, implies average central luminosities of $10^{41-42} \mathrm{ergs} /$ $\mathrm{s}$, well in excess of the observed central $\mathrm{x}$-ray flux of 5 $\times 10^{37} \mathrm{ergs} / \mathrm{s}$ (Fabbiano 1989), or our own limit on a nonthermal optical source of $L<3 \times 10^{37} \mathrm{ergs} / \mathrm{s}$. As Goodman \& Lee (1989) note, however, it is quite possible that this energy release is episodic rather than steady, and argue that the luminosity problem is not fatal, given the large uncertainties in mass transport mechanisms.

\subsection{The Core Model}

If the core of M32 comprises only stars and compact stellar-mass remnants with no black hole, then it will be vulnerable to collapse. If we assume that the dispersion is isotropic with $\sigma=r_{\mathrm{c}} \sqrt{4 \pi G \rho_{0} / 9}$ (Binney \& Tremaine 1987), and that all objects are of about one solar mass, then

$$
\begin{aligned}
t_{\mathrm{r}}= & 6.5 \times 10^{7}\left(\frac{r_{\mathrm{c}}}{0.37 \mathrm{pc}}\right)^{3} \\
& \times\left(\frac{\rho_{0}}{4 \times 10^{6} \mathscr{M}_{\odot} \mathrm{pc}^{-3}}\right)^{1 / 2}\left[\frac{13}{\ln (0.4 N)}\right] \mathrm{yr} .
\end{aligned}
$$

Single-mass models undergo collapse at $\sim 16 t_{\mathrm{r}}$ (Cohn 1980), but more realistic multimass models collapse much sooner, after only a few $t_{\mathrm{r}}$ (Inagaki \& Saslaw 1985).

The relaxation time could be lengthened in an ad hoc manner by increasing the core mass and velocity dispersion, but the requirements are both extreme and implausible. Suppose that the core is dominated by heavy dark stellar remnants of mass $\mathscr{M}_{\mathrm{r}}$. Since $t_{\mathrm{r}} \propto \sigma r_{\mathrm{c}}^{2} / \mathscr{M}_{\mathrm{r}}$, at fixed $r_{\mathrm{c}}$ one would have to increase $\sigma$ by a factor $\geqslant 20$ to $1200 \mathrm{~km} / \mathrm{s}$ at 0 . 1 , to lengthen $t_{\mathrm{r}}$ to a Hubble time. It is totally unclear, however, how to produce such a collection of "hot" yet centrally concentrated dark remnants. The natural mechanism is mass segregation, but a short relaxation time is required for this to be effective; further mass segregation typically leads to lower velocity dispersion for the massive components, not higher (Murphy et al. 1990; Quinlan \& Shapiro 1990). We conclude that a core in M32 cannot be stable against collapse.

An unstable core need not be an argument against the plausibility of the core vs cusp picture if the core can expand after collapse. However, since we infer that $t_{\mathrm{r}}$ is two orders of magnitude less than the Hubble time, it is not likely that we are seeing the core in a state of free expansion from a collapse long ago (Goodman \& Lee 1989). A more likely possibility, given the high mass of $\mathrm{M} 32$, is an oscillating core (Murphy et al. 1990) that is now seen in an interbounce phase. Reversing core collapse, however, requires an effective energy input mechanism that "turns on" at the high densities within a collapsing core. Although this problem has been well studied for globular clusters, we emphasize that the much higher "background" velocity dispersion in M32 greatly enhances the role of stellar collisions, which in turn changes the conditions at core collapse. The main question is if runaway stellar coalescence and formation of a black hole will occur before core collapse can be reversed by energy input from binary formation and stellar evolution.

Stellar collisions would be important even if the M32 core were stable, and become even more important as the core stellar density increases during collapse. For the core model, Eq. (4) gives

$$
\begin{aligned}
t_{\mathrm{c}}= & 1.1 \times 10^{10}\left(\frac{\rho_{0}}{4 \times 10^{6} \mathscr{M}_{\odot} \mathrm{pc}^{-3}}\right)^{-1 / 2}(1+f)^{3 / 2} \\
& \times(1+f / 2)^{-1} \mathrm{yr},
\end{aligned}
$$

where $f$ is the ratio of the mass contained in any dark component relative to the stellar component, which itself is specified by $\rho_{0}$. Here we have assumed solar radii for the stars, that the gravitational focusing term in Eq. (4) dominates, and that stars may collide with both other stars and compact objects but that the compared objects themselves have negligible radii. Clearly in this model, an impressive fraction of the objects in the core will have suffered physical collisions if the core is as old as the universe.

Stellar collisions in the M32 core are likely to produce more massive stars that will remain in the core. The collision simulations of Benz \& Hills (1987) would indicate that the M32 velocity dispersion of $60 \mathrm{~km} / \mathrm{s}$ is too high for physical collisions to make tidal-capture binaries but low enough that stars merge quietly with little mass loss. The short-term effect is to remove kinetic energy but no mass from the core, which will hasten core collapse; this is balanced against more rapid stellar evolution of the remnant, leading to significant mass ejection from the core if the collapse takes place slowly enough.

The structural evolution of galactic nuclei similar to that in M32 has been studied by Quinlan \& Shapiro (1990). The Quinlan and Shapiro simulations show that nuclear collapse can be reversed by stellar evolutionary mass loss if the relaxation time exceeds $10^{8} \mathrm{yr}$, or by formation of a "3-body" binary if the nuclear velocity dispersion is low enough. If a binary does form, however, a num- 
ber of merging generations will already have taken place, and the binary progenitors will be drawn from the merger products; a similar effect is also seen in globular cluster evolution (Lee 1987). The interplay between binary formation and stellar collisions can be seen as follows: The timescale for creation of a single 3-body binary within the core is

$$
\begin{aligned}
t_{3}= & 3.4 \times 10^{12}\left(\frac{n}{4 \times 10^{6} \mathrm{pc}^{-3}}\right)^{-3 / 2}\left(\frac{\mathscr{M}}{\mathscr{M}_{\odot}}\right)^{-7 / 2} \\
& \times\left(\frac{\sigma}{60 \mathrm{~km} / \mathrm{s}}\right)^{6} \mathrm{yr}
\end{aligned}
$$

(Spitzer 1987). In reality, the binary is most likely to be formed at the high densities associated with deep collapse when the core of the system comprises only $\sim 30$ stars (Goodman 1989); however, the timescale for collisions will be much shorter at that point as well. At densities closer to that at typical maximum collapse, the ratio of the binary formation to the collision timescale is

$$
t_{3} / t_{\mathrm{c}}=20\left(\frac{\sigma}{60 \mathrm{~km} / \mathrm{s}}\right)^{5}\left(\frac{n}{10^{9} \mathrm{pc}^{-3}}\right)^{-1 / 2}\left(\frac{\mathscr{M}}{\mathscr{M}_{\odot}}\right)^{-5 / 2} \text {. }
$$

While the quantitative evaluation of Eq. (11) may be highly uncertain, its qualitative behavior shows two interesting results. First, for typical solar mass stars, collisions are highly likely to modify the central population prior to reversal of collapse; but second, the binary is much more likely to form in such a population of massive merger remnants.

The simulations of Quinlan and Shapiro indeed appear to show that the dividing line between runaway collapse and core bounce is related to the initial velocity dispersion of the system, as would be expected from Eq. (11). Formation of a central black hole happens in nuclei dense and "hot" enough to permit several generations of stellar merging and consequential formation of high-mass stars to occur prior to binary formation. The black hole itself would be formed as the evolutionary endpoint of a high-mass star, and then grow by subsequent mass accretion.

The simulations of Quinlan and Shapiro would suggest that the M32 core appears to be close to the dividing line between systems that will eventually bounce and those that will suffer irreversible collapse. In such a marginal state, uncertainties in the models become highly significant and at present we cannot resolve this issue. However, in all models regardless of the final outcome, the average stellar mass at the very core will be substantially increased by mergers. We thus conclude that unless we are seeing the core at a special time, a core without a black hole should have collapsed on at least one occasion, with concomitant strong mass segregation and modification of the central population. If the collapse reversed without formation of a black hole, significant modification of the population by stellar mergers should still continue.

The Quinlan and Shapiro models begin with only one mass component present originally and also set the age of the merged stars to zero, regardless of the state of their progenitors. Clearly, understanding mass loss and evolu- tion of stars in a system in which collisions are significant is important for future work. Primordial binaries may be important in controlling core collapse, but at this time little can be said beyond speculation on what role they would play in M32. It must also be remembered that the core of M32 is not an isolated cluster, but is merely the denser part of a system in which relaxation effects may still be important out to larger radii. At the very least, expansion of the core after collapse may be limited by interaction with stars outside the core, perhaps hastening recollapse. Last, we note that if the M32 core can bounce, given the profound changes in the core population over the event, conditions for future collapse will be strongly different and may of themselves lead to an irreversible collapse.

We now consider whether the observations can support the core collapse picture. Photometrically, the existence of a core or even a cusp (that we have considered above as a signature of a black hole) is readily matched by core collapse models. The multimass globular cluster models of Chernoff \& Weinberg (1990) show that the various mass components, each containing objects of mass $\mathscr{M}_{k}$, reach equipartition during collapse with logarithmic slopes in space density given by

$$
\gamma_{k}=-0.23\left(8.2 \frac{\mathscr{M}_{f}}{\mathscr{M}_{u}}+1.5\right)
$$

where $\mathscr{M}_{u}$ is the mass of the heaviest remnant. A visibleto-dark remnant mass ratio $\mathscr{M}_{k} / \mathscr{M}_{u} \approx 0.6$, for example, would generate a $\gamma \approx-1 / 2$ cusp. A more difficult problem, however, is that there is little evidence for any unusual population at the center of M32 suggestive of the stellar merging that we expect to occur under this picture. Lugger et al. (1992) find $B-R$ constant to $0.01 \mathrm{mag}$ into the core. Neither Lugger et al. nor the present observations show any evidence of unusual photometric structures in the galaxy or departures of the isophotes from ellipses. In this context, it is interesting that Nieto et al. (1992) have raised the possibility that the centralmost AGB stars that they detect in M32 may in part form a disky subsystem. Nieto et al. argue for a past episode of gaseous dissipation and star formation. Stellar collisions are also a dissipative process, however; and given the strong rotation in M32 some of the collision products should be more closely confined to the central plane than the parent population. A single generation of stellar mergers is likely to make "blue stragglers" that will later evolve into AGB stars. The subtle effect found by Nieto et al. may be more evocative of the low but significant collision frequency within a black hole cusp. In contrast, the wholesale merging that would take place in the core model appears to be ruled out by the constant central isophote ellipticity as well as the lack of a color gradient. This also suggests that if a central black hole was formed in a nonreversible core collapse, then this must have happened long enough ago for stellar evolution to have erased all evidence of the massive stars that would be generated by such an event. 


\section{CONCLUSIONS}

The new HST observations presented here show that the M32 nucleus is extremely dense, with $\rho_{0} \geqslant 4 \times 10^{6} \mathscr{M}_{\odot} \mathrm{pc}^{-3}$ if M32 has a core with $r_{\mathrm{c}} \leqslant 0.37 \mathrm{pc}$, and $\rho_{0}>3 \times 10^{7}$ $\mathscr{M}_{\odot} \mathrm{pc}^{-3}$ if it instead has a central $r^{-1 / 2}$ cusp. Limits on the M32 core radius are nearly three times smaller, and those on the central density at least four times greater, than values inferred from the best ground-based observations; the core is still unresolved at even $H S T$ resolution.

The stellar cusp is consistent with an $\mathscr{M}_{\bullet}=2.8 \times 10^{6}$ $\mathscr{M}_{\odot}$ black hole, and evolves only slowly over the age of the universe owing to relaxation effects and stellar collisions. In contrast, the core model without a black hole is highly vulnerable to collapse and modification of its population by stellar mergers. The new core parameters presented here imply a central relaxation that is almost an order of magnitude shorter than previous estimates; a shorter stellar collision time is also implied. The present state of theory cannot show whether such a core must generate a massive central black hole, or whether it can rebound after col- lapse, perhaps to go through several later collapse cycles. Simple calculations show that stellar mergers play a central role in such a collapsing core; resolution of the fate of the M32 core perhaps lies as much in the field of stellar interiors as stellar dynamics. At the very least, collapse induced changes in the central population will make any core bounce highly time asymmetric. The lack of a central color gradient as well as the constant central isophote shape in the presence of strong rotation may argue that core collapse has not recently occurred. The HST observations raise the intriguing possibility that M32 may be the first example of a site where structural evolution of a gravitating system ends with formation of a massive black hole.

We thank Dr. Jeremy Goodman for useful conversations. This research was conducted by the WFPC Investigation Definition Team, supported in part by NASA Grant No. NAS5-1661.

\section{REFERENCES}

Benz, W., \& Hills, J. G. 1987, ApJ, 323, 614

Binney, J., \& Tremaine, S. 1987, Galactic Dynamics (Princeton University Press, Princeton)

Burrows, C., Holtzman, J. A., Faber, S. M., Bely, P., Husan, H., Lynds, C. R., \& Schroeder, D. 1991, ApJ, 369, L21

Burstein, D. 1989, private communication

Chernoff, D. F., \& Weinberg, M. D. 1990, ApJ, 351, 121

Cohn, H. 1980, ApJ, 242, 765

Dressler, A., \& Richstone, D. O. 1988, ApJ, 324, 701

Fabbiano, G. 1989, ARA\&A, 27, 87

Goodman, J. 1989, in Dynamics of Dense Stellar Systems, edited by D.

Merritt (Cambridge University Press, Cambridge), p. 183

Goodman, J., \& Lee, H. M. 1989, ApJ, 337, 84

Griffiths, R. 1989, Wide Field and Planetary Camera Instrument Handbook (STScI publication, Baltimore)

Inagaki, S., \& Saslaw, W. C. 1985, ApJ, 292, 339

Kent, S. M. 1983, ApJ, 266, 562

Lauer, T. R. 1985, ApJ, 292, 104

Lauer, T. R. 1989, PASP, 101, 445

Lee, H. M. 1987, ApJ, 319, 801

Lee, M. H., \& Goodman, J. 1989, ApJ, 343, 594
Lucy, L. B. 1974, AJ, 79, 745

Lugger, P. M., Cohn, H. N., Cederbloom, S. E., Lauer, T. R., \& McClure, R. D. 1992, AJ 104, 83

Michard, R., \& Nieto, J. L. 1991, A\&A, 243, L17

Murphy, B. W., Cohn, H. N., \& Durisen, R. H. 1989, in Dynamics of Dense Stellar Systems, edited by D. Merritt (Cambridge University Press, Cambridge), p. 97

Murphy, B. W., Cohn, H. N., \& Hut, P. 1990, MNRAS, 245, 335

Nieto, J. L., Bacon, R., Emsellem, E., \& Monnet, G. 1992, A\&A submitted

Peterson, C. J., \& King, I. R. 1975, AJ, 80, 427

Quinlan, G. D., \& Shapiro, S. L. 1990, ApJ, 356, 483

Richardson, W. H. 1972, J. Opt. Soc. A, 62, 52

Richstone, D. O., Bower, G., \& Dressler, A. 1990, ApJ, 353, 118

Spitzer, L. 1987, Dynamical Evolution of Globular Clusters (Princeton University Press, Princeton)

Tonry, J. L. 1984, ApJ, 283, L27

Tonry, J. L. 1987, ApJ, 322, 632

Tonry, J. L., \& Schneider, D. P. 1988, AJ, 96, 807

Young, P. J. 1980, ApJ, 242, 1232 


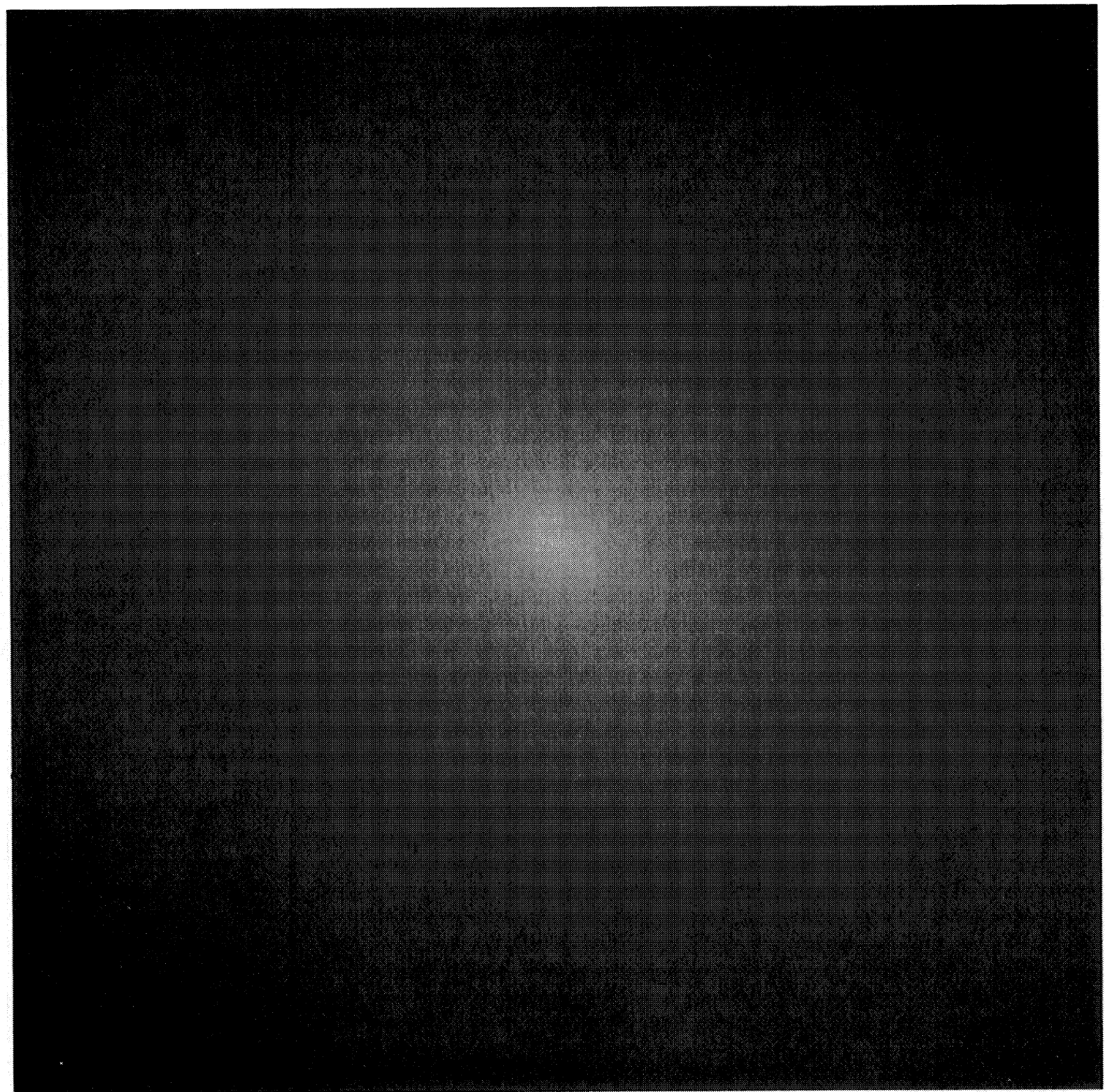

FIG. 1. 300 s F555W PC image of the central 4.00×4"00 region of M32. The stretch is logarithmic and set so that black to white covers a range of 100 in surface brightness.

Lauer et al. (see page 554) 


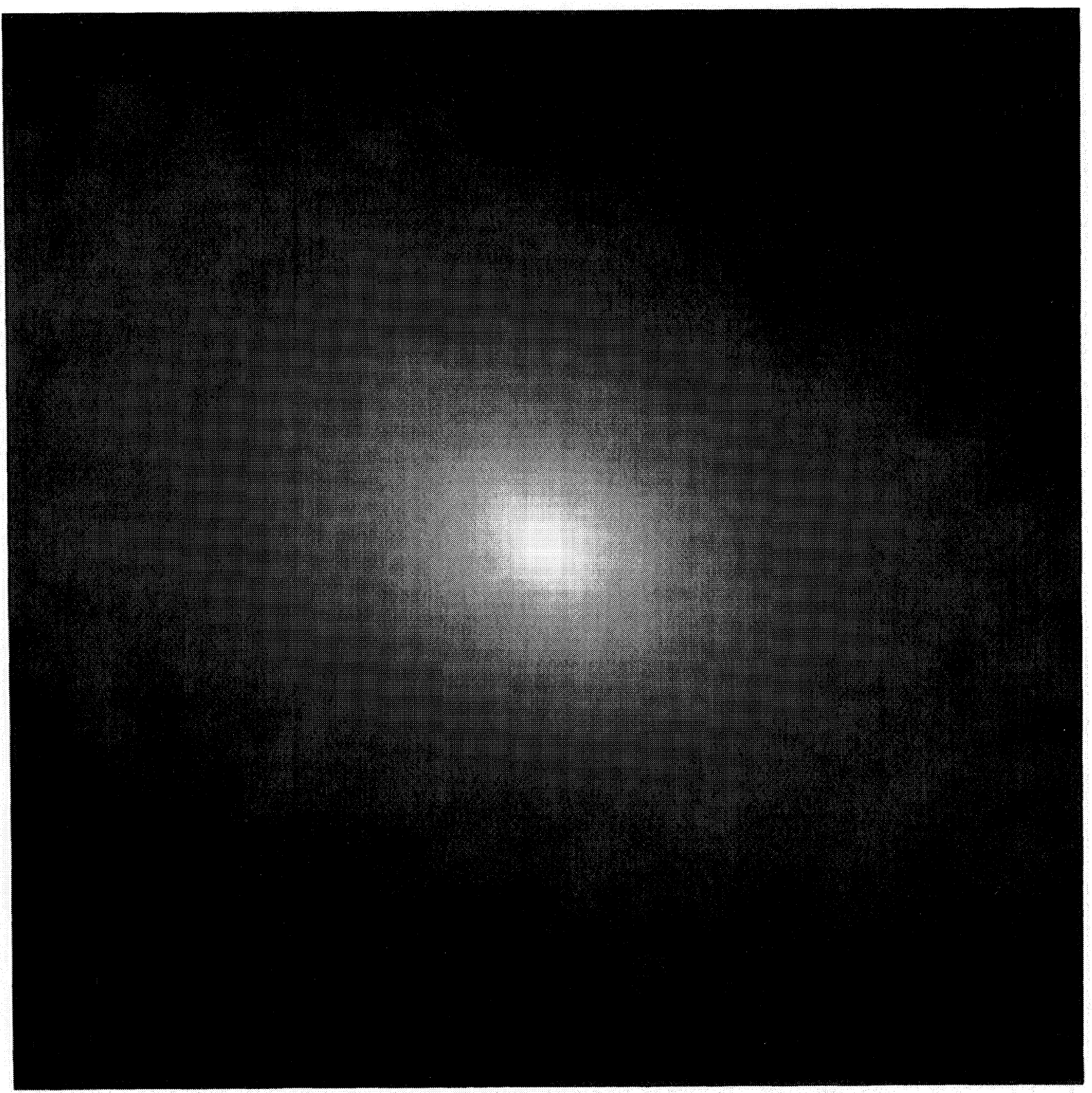

FIG. 2. Lauer et al. (see page X) Deconvolved image of the M32 center after 160 iterations of Lucy-Richardson deconvolution. The region of the image shown and the stretch is the same as in Fig. 1.

Lauer et al. (see page 554) 


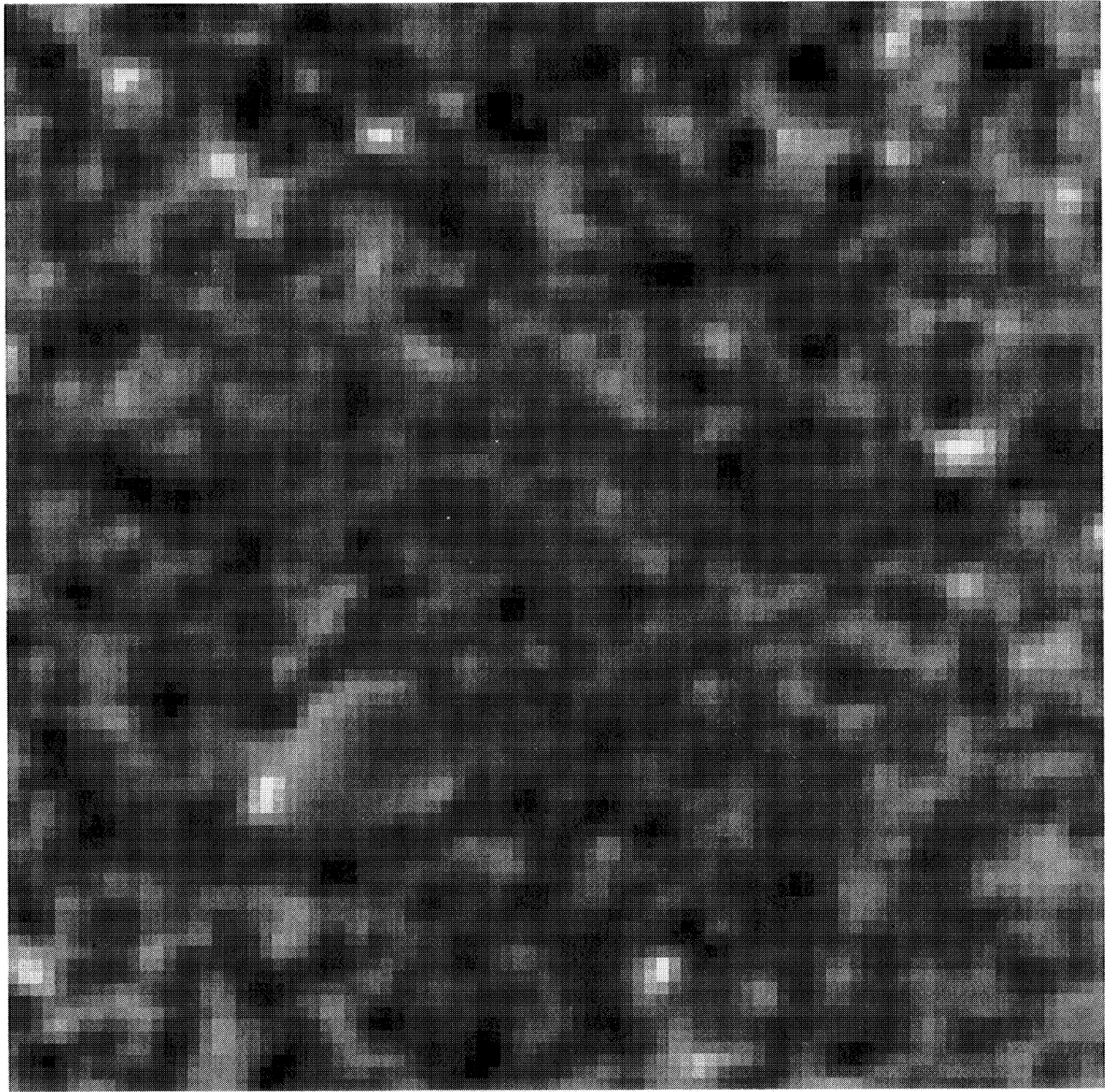

FIG. 3. Lauer et al. (see page $\mathrm{X}$ ) The deconvolved image divided by a model reconstructed from the deconvolved surface photometry profiles. The rms fluctuation over the region shown is $0.05 \mathrm{mag}$ in excellent agreement with luminosity fluctuation amplitude expected from the parameters given by Tonry \& Schneider (1988). The apparent decrease in relative noise near the center is real and is due to the greater surface brightness there. The large smooth feature offset from the nucleus is a region where a flatfield defect was filled in prior to deconvolution. 